\title{
Proliferation and differentiation of human osteoblasts from a type 2 diabetic patient in vitro
}

\author{
J. Li ${ }^{1,2}$, C.Y. Liu ${ }^{1}$, Y.F. Jiang ${ }^{1}$, X.Z. Wei ${ }^{1}$ and J.U. Li ${ }^{1,3}$ \\ ${ }^{1}$ Department of Dental Implant Center, Beijing Stomatological Hospital, \\ School of Stomatology, Capital Medical University, Beijing, China \\ ${ }^{2}$ Department of Stomatology, Tangshan People's Hospital, Tangshan, China \\ ${ }^{3}$ Beijing Key Laboratory of Tooth Regeneration and Function Reconstruction, \\ Capital Medical University, Beijing, China
}

Corresponding author: J.U. Li

E-mail: lijunj1@126.com

Genet. Mol. Res. 14 (3): 11292-11299 (2015)

Received March 3, 2015

Accepted June 8, 2015

Published September 22, 2015

DOI http://dx.doi.org/10.4238/2015.September.22.23

\begin{abstract}
We investigated the proliferation and differentiation potential of human osteoblasts from a type 2 diabetic patient in vitro. Human osteoblasts were obtained from a healthy subject and a type 2 diabetic patient and were cultured in vitro using the tissue explant adherent method. Differences in cell morphology were observed under a phase contrast microscope. The osteogenic differentiation capacity was evaluated by alizarin red staining, 3-(4,5-dimethylthiazol-2-yl)2,5-diphenyltetrazolium bromide (MTT) assay, alkaline phosphatase (ALP) staining, and detection of bone Gla-protein (BGP) and Col1. Expression of Runx-2 and Col-1 was detected using RT-PCR and western blot. Our data indicated that alveolar bone osteoblasts from the type 2 diabetic patient exhibited poorer growth, smaller calcium nodule formation, slower proliferation, and lower ALP, BGP, and Col-1 concentrations in the cell culture supernatant, as compared to control
\end{abstract}


cells $(\mathrm{P}<0.05)$. Combined, our study indicated that alveolar bone osteoblasts from a type 2 diabetic patient exhibited slower proliferation and decreased differentiation, as compared to healthy control, when cultured in vitro.

Key words: Type 2 diabetes; Alveolar bone; Osteoblast; Osteoblast proliferation and differentiation

\section{INTRODUCTION}

Diabetes is a systemic metabolic disorder (Kinane and Marshall, 2001). Most patients have type 2 diabetes mellitus (T2DM) (Hasegawa et al., 2008; L'Heveder and Nolan, 2013). Recent studies showed that diabetes can damage the balance of bone metabolism (McCracken et al., 2000). Furthermore, clinical studies showed that the failure rate of dental implants in diabetic patients with missing teeth is higher than in healthy controls (Fiorellini et al., 2000; Bugea et al., 2008); however, the mechanism remains unclear. Lack of insulin in diabetic patients can reduce the number of osteoblasts and the synthesis of bone matrix (Tervonen and Oliver, 1993; Taylor, 2001). Additionally, sustained hyperglycemia may also inhibit osteoblast differentiation (Santana et al., 2003). However, the formation and differentiation of osteoblasts play a key role in bone growth and repair. Thus, evaluation of the biological characteristics of osteoblasts from diabetic patients is necessary to understand the mechanism by which diabetes affects bone metabolism.

This study aimed to establish an in vitro osteoblast culture system and compare the proliferation and differentiation of osteoblasts derived from diabetic patients and healthy humans to better understand the absorption and regeneration of alveolar bone and its regulatory mechanisms.

\section{MATERIAL AND METHODS}

\section{Ethical statement}

Ethical approval was granted by the Ethics Committee of Beijing Stomatological Hospital. Patients were provided with a written information letter about the study, and participants provided their written informed consent to participate in the study.

\section{Human alveolar bone osteoblast isolation and culture}

Alveolar bone debris from oral implants in healthy humans and a T2DM patient were used in this study. The patient was diagnosed with type 2 diabetes more than 2 years prior, and had no other serious diseases, complications, inflammation, or recent operations. The bone debris was obtained during the preparation of a planting nest during an aseptic operation. The obtained bone debris was washed with 1X PBS with double antibiotics $(100 \mathrm{~g} / \mathrm{mL}$ penicillin and streptomycin) and centrifuged 3 times. Cells were cultured in $\alpha$-minimal essential medium containing $10 \%$ fetal bovine serum at $37^{\circ} \mathrm{C}$ with $5 \% \mathrm{CO}_{2}$. The cells were passaged when the cell density reached approximately $80 \%$. 


\section{Alizarin red staining}

Changes in cell growth and morphology were observed under an inverted microscope. The culture medium was discarded after incubation for 21 days, and the cells were washed with PBS 2 times. The cells were fixed with 4\% paraformaldehyde for $20 \mathrm{~min}$ and stained with alizarin red for $10 \mathrm{~min}$. The formation of calcium nodules was observed and pictures were taken.

\section{Alkaline phosphatase (ALP) chemical staining and quantitative detection}

ALP was stained and detected using the ALP staining kit and quantitative detection kit according to the manufacturer protocols. Fourth generation osteoblasts from healthy humans and patients with T2DM were digested and cultured on a 48-well plate, and the optical density (OD) value at a wavelength of $520 \mathrm{~nm}$ was determined.

\section{Detection of cell proliferation by 3-(4,5-dimethylthiazol-2-yl)-2,5- diphenyltetrazolium bromide (MTT) assay}

Cells were cultured on 96-well plates and measured over 11 continuous days. MTT $\left(20 \mu \mathrm{L}, 5 \mathrm{mg} / \mathrm{mL}\right.$ ) was added to the cells and was incubated for $4 \mathrm{~h}$ at $37^{\circ} \mathrm{C}$. The culture medium was discarded, and $100 \mathrm{~mL}$ dimethylsulfoxide was added to each well. After shaking at low speed for $10 \mathrm{~min}$, the MTT solution was aspirated and the OD values of the supernatant were read at $490 \mathrm{~nm}$ using a microplate reader. The experiments were repeated 3 times and a growth curve was obtained.

\section{Bone Gla-protein (BGP) concentration determination}

Cells were treated as described above and the culture supernatants were collected on days 3, 7, and 9. The BGP concentration was determined using the 125I osteocalcin RIA Kit following the manufacturer protocol. The sedimentation cell per milliliter number for each tube was measured with a $\gamma$ counter, and the BGP concentration was determined according to a standard curve.

\section{Col-1 concentration determination}

Cells were treated as described above and the culture supernatants were collected on days 3, 7, and 9. The Col-1 concentration was determined using the Col-1 enzyme-linked immunosorbent assay (ELISA) kit. OD values were read at $450 \mathrm{~nm}$ using a microplate reader.

\section{Evaluation of Runx-2 and Col-1 levels by real-time PCR and western blot}

Total RNA was extracted using the RNeasy Mini Kit (Qiagen, Germantown, MD, USA) according to the manufacturer protocol. Total RNA $(1 \mu \mathrm{g})$ was subjected to reverse transcription using a reverse transcription system (Promega, Madison, WI, USA). Real-time PCR was performed using an RT-PCR kit (Takara, Shiga, Japan). $\beta$-actin gene expression was used as an internal control for normalization. The PCR 
products underwent electrophoresis on a $1.0 \%$ agarose gel and were visualized under UV illumination using ethidium bromide staining. Primer sequences were as follows: $\beta$-actin F: 5'-TGGCACCCAGCACAATGAA-3', R: 5'-CTAAGTCATAGTCCGCCTAGAAGCA-3'; Runx2 F: 5'-CACTGGCGCTGCAACAAGA-3', R: 5'-CATTCCGG AGCTCAGCAGAATAA-3'; Col1 F: 5'-TCCACATACCTTTATTCCAGGAATC-3', R: 5'-CCCGGGTTTAGAGACAACTTC-3'.

Cell lysates were separated by sodium dodecyl sulfate polyacrylamide gel electrophoresis. The separated proteins were electrotransferred to polyvinylidene fluoride membranes. The membranes were used for immunoblotting with the required antibodies. The protein bands were scanned and quantified using the ratio to $\beta$-actin.

\section{Statistical analyses}

The data were analyzed with the SPSS 19.0 software. The results are reported as means $\pm \mathrm{SD}$. The $t$-test was used to evaluate differences between groups. A P value less than 0.05 was considered to be statistically significant.

\section{RESULTS}

\section{Cell culture and observation of osteoblasts in vitro}

In the control group, cells grew out of the tissue blocks 5 days after culture. Morphologically, the cells were irregular strips or spindles. The cell density reached approximately $80 \%$ after 14 days in culture. In the T2DM group, cells grew out of the tissue blocks after 8 days in culture. The cellular morphology was similar to the control group. The T2DM cells reached $80 \%$ confluence on day 21 . Calcium nodules in the T2DM group were smaller than that of the control group (Figure 1).

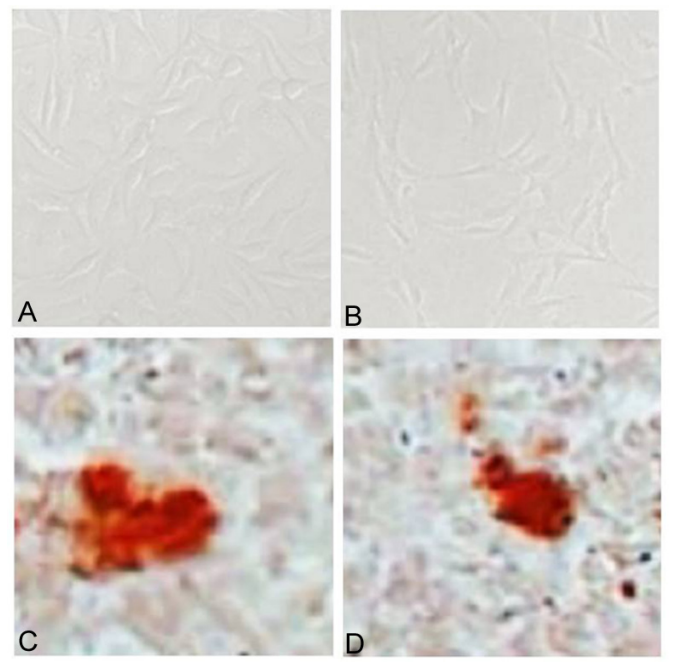

Figure 1. Osteoblast morphology and calcified nodules (100X). A. Osteoblasts from human alveolar bone in the control group. B. Osteoblasts from human alveolar bone in the T2DM group. C. Alizarin red staining of osteoblasts from the control group. D. Alizarin red staining of osteoblasts from the T2DM group. 


\section{ALP detection}

In the control group, many positive ALP cells were observed after staining, with deep staining observed in the cytoplasm. Fewer ALP-stained cells were obtained in the T2DM group, and the cytoplasm was pale. Quantitative detection of ALP showed that ALP was higher in the control group than in the T2DM group (Figure 2).
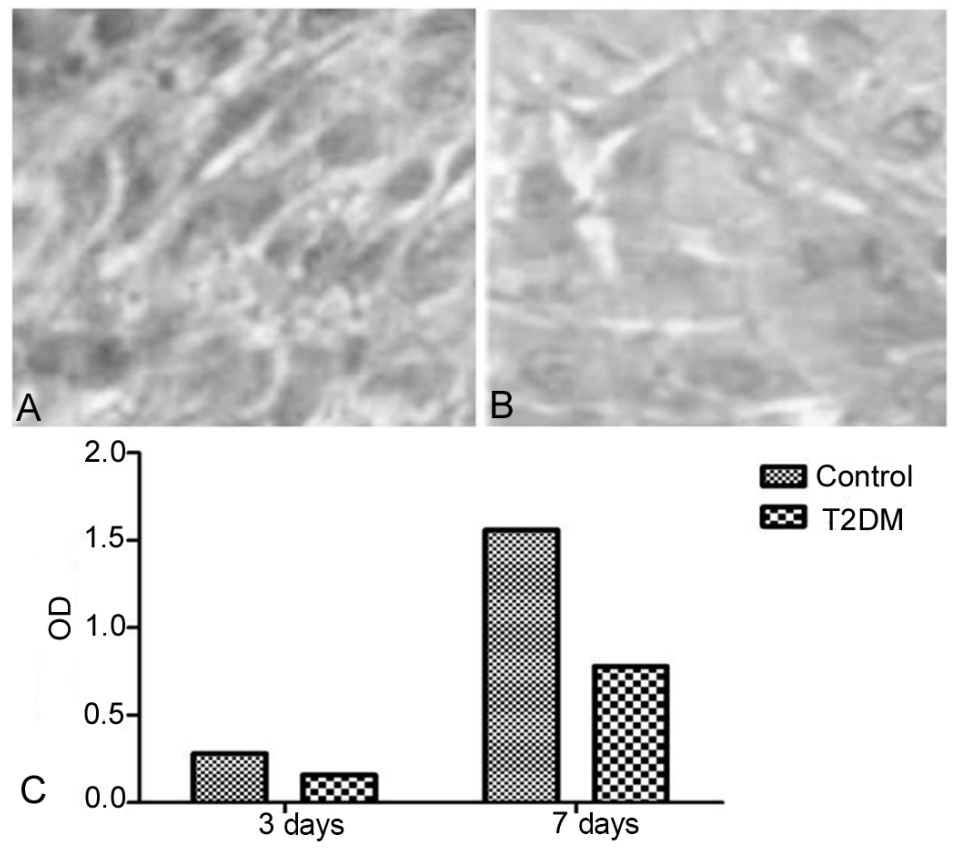

$\notin$ Control

$\infty$ T2DM

Figure 2. Alkaline phosphatase (ALP) detection. A. ALP staining in the control group. B. ALP staining in the T2DM group. C. Quantitative detection of ALP.

\section{Proliferation of osteoblasts in vitro}

Cell growth was determined by MTT assay (Figure 3). Proliferation was greatest on the seventh day in the control group and gradually reduced after 10 days. Proliferation was greater in the control group than in the T2DM group at all times.

\section{BGP and Col-1 concentration determination}

The concentration of BGP and Col-1 in the control group was greater than in the T2DM group $(\mathrm{P}<0.05$; Tables 1 and 2$)$.

\section{Runx-2 and Col-1 expression}

Runx-2 and Col-1 expressed in both groups. However, the expression levels were greater in the control group than in the T2DM group $(\mathrm{P}<0.05$; Table 3 and Figure 4). 


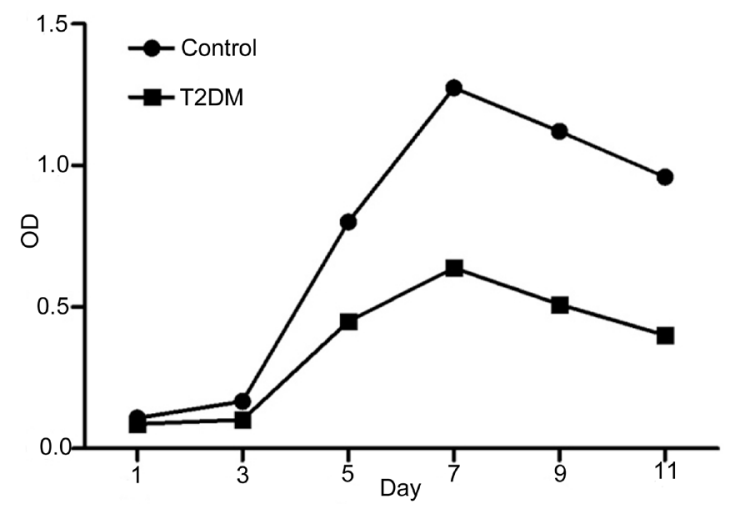

Figure 3. Cell growth curves.

Table 1. Bone Gla-protein (BGP) content in osteoblast culture supernatants $(\mathrm{N}=3$, means $\pm \mathrm{SE})$.

\begin{tabular}{lccc}
\hline Group & \multicolumn{3}{c}{ BGP $(\mathrm{ng} / \mathrm{mL})$} \\
\cline { 2 - 4 } & 3 days & 7 days & 9 days \\
\hline Control & $6.32 \pm 0.04$ & $12.95 \pm 0.07$ & $10.48 \pm 0.11$ \\
T2DM & $3.20 \pm 0.06^{*}$ & $9.87 \pm 0.15^{*}$ & $8.04 \pm 0.07^{*}$ \\
\hline$* \mathrm{P}<0.05$ & & &
\end{tabular}

$* \mathrm{P}<0.05$.

Table 2. Col-1 content in osteoblast culture supernatants $(\mathrm{N}=3$, means $\pm \mathrm{SE})$.

\begin{tabular}{lccc}
\hline Group & \multicolumn{3}{c}{ Col-1 $(\mu \mathrm{g} / \mathrm{L})$} \\
\cline { 2 - 4 } & 3 days & 7 days & 9 days \\
\hline Control & $4.88 \pm 0.42$ & $10.02 \pm 0.14$ & $7.28 \pm 0.16$ \\
T2DM & $3.74 \pm 0.01^{*}$ & $5.07 \pm 0.23^{*}$ & $3.29 \pm 0.46^{*}$ \\
\hline
\end{tabular}

$* \mathrm{P}<0.05$

Table 3. Runx-2 and Col-1 expression levels $(\mathrm{N}=3$, means $\pm \mathrm{SE})$.

\begin{tabular}{lll}
\hline Group & Runx-2 & Col-1 \\
\hline Control & $1.576 \pm 0.21$ & $1.469 \pm 0.27$ \\
T2DM & $0.466 \pm 0.03 *$ & $0.580 \pm 0.41 *$ \\
\hline$* \mathrm{P}<0.05$. & &
\end{tabular}

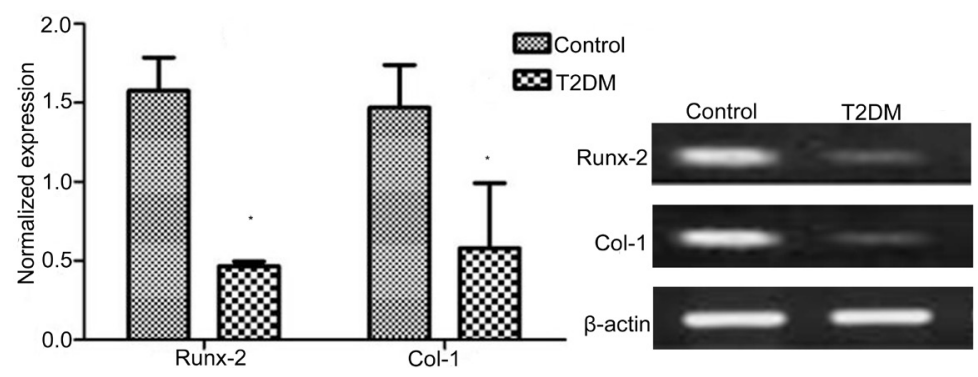

Figure 4. Expression levels of Runx-2 and Col-1. A. RT-PCR results. B. Western blotting results. 


\section{DISCUSSION}

Alveolar bone is the most active in human bone and its state has a direct impact on oral health and the function of the stomatognathic system (Heitz-Mayfield, 2005; Holmlund et al., 2006).

Alveolar bone osteoblasts originate from pluripotent bone marrow stromal cells. They are involved in the synthesis and secretion of bone matrix and contribute to matrix mineralization, thereby forming bone tissue (Krentz and Bailey, 2005; Mellado-Valero et al., 2007). It has been reported in animal and clinical experiments that osteoblasts can differentiate and form new bone (Nevins et al., 1998; Zimmet et al., 2003; Seo et al., 2004). Therefore, culturing osteoblasts in vitro is of interest for bone tissue engineering. T2DM is a metabolic disorder. Studies have shown that the alveolar bone of rats with T2DM is more severely damaged than normal rats (Wang et al., 2010). Sustained hyperglycemia may reduce ALP and delay mineralization (Chen, 2004; Simao et al., 2007). Damage to the alveolar bone is more serious in patients with poor glycemic control than in healthy controls (Lalla et al., 2000; Gevorgyan et al., 2007). However, the mechanisms of this effect remain unclear.

In this study, we cultured alveolar bone osteoblasts from T2DM patients and healthy humans in vitro. We found that there are differences in ALP activity, cellular growth state, proliferation, and differentiation between groups. The alveolar bone osteoblasts from T2DM patients grew slowly and displayed less calcium precipitation by alizarin red staining, indicating that osteoblasts from T2DM patients have weak osteogenic ability in vitro. Osteoblast proliferation and ALP activity in T2DM patients were also weaker than in healthy humans.

Runx-2 and Col-1 are early osteogenic genes, and the expression of Runx-2 is the most specific indicator of bone formation (Lind et al., 1995; Takeda et al., 2001). Runx-2 is upstream of Col-1. The Runx-2 and Col-1 mRNA levels in the T2DM group were lower than in normal humans, suggesting that diabetes reduced osteogenic differentiation. BGP and Col1 are protein frameworks that compose bone tissue, and could be specific markers for bone tissue formation (Sanguineti et al., 2008; Sun et al., 2012). The secretion of BGP and Col-1 in osteoblasts from T2DM patients was lower than in healthy human.

These results showed that proliferation, differentiation, and bone matrix synthesis were decreased in osteoblasts from T2DM patients, as compared to healthy humans, suggesting that diabetes affects alveolar bone metabolic balance. Insulin deficiency or sustained hyperglycemia could inhibit the synthesis and secretion of bone matrix, thus altering peri-implant bone bonding.

Studies on the biological characteristics of osteoblasts of T2DM can provide insight into the mechanism by which diabetes affects bone metabolism, thereby improving the success rate of dental implants in T2DM patients.

\section{ACKNOWLEDGMENTS}

We thank all colleagues in the Beijing Key Laboratory of Tooth Regeneration and Function Reconstruction.

\section{REFERENCES}

Bugea C, Luongo R, Di Iorio D, Cocchetto R, et al. (2008). Bone contact around osseointegrated implants: histologic analysis of a dual-acid-etched surface implant in a diabetic patient. Int. J. Periodontics Restorative Dent. 28: 145-151. 
Chen TL (2004). Inhibition of growth and differentiation of osteoprogenitors in mouse bone marrow stromal cell cultures by increased donor age and glucocorticoid treatment. Bone 35: 83-95.

Fiorellini JP, Chen PK, Nevins M and Nevins ML (2000). A retrospective study of dental implants in diabetic patients. Int. J. Periodontics Restorative Dent. 20: 366-373.

Gevorgyan A, La Scala GC, Sukhu B, Leung IT, et al. (2007). An in vitro model of radiation-induced craniofacial bone growth inhibition. J. Craniofac. Surg. 18: 1044-1050.

Hasegawa H, Ozawa S, Hashimoto K, Takeichi T, et al. (2008). Type 2 diabetes impairs implant osseointegration capacity in rats. Int. J. Oral Maxillofac. Implants 23: 237-246.

Heitz-Mayfield LJ (2005). Disease progression: identification of high-risk groups and individuals for periodontitis. J. Clin. Periodontol. 32: 196-209.

Holmlund A, Holm G and Lind L (2006). Severity of periodontal disease and number of remaining teeth are related to the prevalence of myocardial infarction and hypertension in a study based on 4,254 subjects. J. Periodontol. 77 : 1173-1178.

Kinane DF and Marshall GJ (2001). Periodontal manifestations of systemic disease. Aust. Dent. J. 46: 2-12.

Krentz AJ and Bailey CJ (2005). Oral antidiabetic agents: current role in type 2 diabetes mellitus. Drugs 65: 385-411.

L'Heveder R and Nolan T (2013). International Diabetes Federation. Diabetes Res. Clin. Pract. 101: 349-351.

Lalla E, Lamster IB, Feit M, Huang L, et al. (2000). Blockade of RAGE suppresses periodontitis-associated bone loss in diabetic mice. J. Clin. Invest. 105: 1117-1124.

Lind M, Deleuran B, Thestrup-Pedersen K, Soballe K, et al. (1995). Chemotaxis of human osteoblasts. Effects of osteotropic growth factors. APMIS 103: 140-146.

McCracken M, Lemons JE, Rahemtulla F, Prince CW, et al. (2000). Bone response to titanium alloy implants placed in diabetic rats. Int. J. Oral Maxillofac. Implants 15: 345-354.

Mellado-Valero A, Ferrer Garcia JC, Herrera Ballester A and Labaig Rueda C (2007). Effects of diabetes on the osteointegration of dental implants. Med. Oral Patol. Oral. Cir. Bucal. 12: E38-43.

Nevins ML, Karimbux NY, Weber HP, Giannobile WV, et al. (1998). Wound healing around endosseous implants in experimental diabetes. Int. J. Oral Maxillofac. Implants 13: 620-629.

Sanguineti R, Storace D, Monacelli F, Federici A, et al. (2008). Pentosidine effects on human osteoblasts in vitro. Ann. N. Y. Acad. Sci. 1126: 166-172.

Santana RB, Xu L, Chase HB, Amar S, et al. (2003). A role for advanced glycation end products in diminished bone healing in type 1 diabetes. Diabetes 52: 1502-1510.

Seo BM, Miura M, Gronthos S, Bartold PM, et al. (2004). Investigation of multipotent postnatal stem cells from human periodontal ligament. Lancet 364: 149-155.

Simao AM, Beloti MM, Rosa AL, de Oliveira PT, et al. (2007). Culture of osteogenic cells from human alveolar bone: a useful source of alkaline phosphatase. Cell Biol. Int. 31: 1405-1413.

Sun DC, Li DH, Ji HC, Rao GZ, et al. (2012). In vitro culture and characterization of alveolar bone osteoblasts isolated from type 2 diabetics. Braz. J. Med. Biol. Res. 45: 502-509.

Takeda S, Bonnamy JP, Owen MJ, Ducy P, et al. (2001). Continuous expression of Cbfa1 in nonhypertrophic chondrocytes uncovers its ability to induce hypertrophic chondrocyte differentiation and partially rescues Cbfa1-deficient mice. Genes Dev. 15: 467-481.

Taylor GW (2001). Bidirectional interrelationships between diabetes and periodontal diseases: an epidemiologic perspective. Ann. Periodontol. 6: 99-112.

Tervonen T and Oliver RC (1993). Long-term control of diabetes mellitus and periodontitis. J. Clin. Periodontol. 20 : 431-435.

Wang F, Song YL, Li DH, Li CX, et al. (2010). Type 2 diabetes mellitus impairs bone healing of dental implants in GK rats. Diabetes Res. Clin. Pract. 88: e7-9.

Zimmet P, Shaw J and Alberti KG (2003). Preventing Type 2 diabetes and the dysmetabolic syndrome in the real world: a realistic view. Diabet. Med. 20: 693-702. 